\title{
Dynamic Behaviors of a Discrete Periodic Predator-Prey-Mutualist System
}

\author{
Liya Yang, ${ }^{1}$ Xiangdong Xie, ${ }^{2}$ and Fengde Chen ${ }^{2}$ \\ ${ }^{1}$ College of Mathematics and Computer Science, Fuzhou University, Fuzhou, Fujian 350116, China \\ ${ }^{2}$ Department of Mathematics, Ningde Normal University, Ningde, Fujian 352300, China \\ Correspondence should be addressed to Xiangdong Xie; ndsyxxd@163.com and Fengde Chen; fdchen@263.net
}

Received 3 June 2015; Accepted 1 September 2015

Academic Editor: Pavel Rehak

Copyright (c) 2015 Liya Yang et al. This is an open access article distributed under the Creative Commons Attribution License, which permits unrestricted use, distribution, and reproduction in any medium, provided the original work is properly cited.

\begin{abstract}
A nonautonomous discrete predator-prey-mutualist system is proposed and studied in this paper. Sufficient conditions which ensure the permanence and existence of a unique globally stable periodic solution are obtained. We also investigate the extinction property of the predator species; our results indicate that if the cooperative effect between the prey and mutualist species is large enough, then the predator species will be driven to extinction due to the lack of enough food. Two examples together with numerical simulations show the feasibility of the main results.
\end{abstract}

\section{Introduction}

As was pointed out by Berryman [1], the dynamic relationship between predator and prey has long been and will continue to be one of the dominant themes in both ecology and mathematical ecology due to its universal existence and importance. Recently, predator-prey models have been studied widely [2-7]. It brings to our attention that all the works of [27] are dealing with the relationship between two species, while, in the real world, the relationship among species is very complicated and it needs to consider the three-species models. Many scholars [8-13] studied the dynamic behaviors of the three-species models.

Moreover, mutualism is one of the most important relationships in the theory of ecology. Mutualism is a symbiotic association between any two species and the interaction between the two species is beneficial to both of the species [14]. Already, many scholars [15-21] studied the dynamic behaviors of cooperative models. It brings to our attention that although predator-prey and mutualism can be recognized as major issues in both applied mathematics and theoretical ecology, few scholars have considered predator-prey system with cooperation in three species. But this phenomenon really exists in nature. For example, while aphids are preyed by natural enemies, they are protected by some natural friends like ants; there ants eat the honeydew that aphids excrete and help to overcome the resource scarcity of offspring [22, 23].

In 2009, Rai and Krawcewicz [24] proposed the following predator-prey-mutualist system:

$$
\begin{aligned}
& \frac{d x}{d t}=\alpha x\left(1-\frac{x}{K}\right)-\frac{\beta x z}{1+m y}, \\
& \frac{d y}{d t}=\gamma y\left(1-\frac{y}{l x+L_{0}}\right), \\
& \frac{d z}{d t}=z\left(-s+\frac{c \beta x}{1+m y}\right),
\end{aligned}
$$

where $x(t), y(t)$, and $z(t)$ denote the densities of prey, mutualist, and predator population at any time $t$, respectively; they applied the equivariant degree method to study Hopf bifurcations phenomenon of the system.

Recently, Yang et al. [25] argued that, due to seasonal effects of weather, temperature, food supply, mating habits, 
and so forth, a more appropriate system should be a nonautonomous one, and they proposed and studied the following system:

$$
\begin{aligned}
& \dot{x}=x\left(a_{1}(t)-b_{1}(t) x-\frac{c_{1}(t) z}{d_{1}(t)+d_{2}(t) y}\right), \\
& \dot{y}=y\left(a_{2}(t)-\frac{y}{d_{3}(t)+d_{4}(t) x}\right), \\
& \dot{z}=z\left(-a_{3}(t)+\frac{k_{1}(t) c_{1}(t) x}{d_{1}(t)+d_{2}(t) y}-b_{2}(t) z\right) .
\end{aligned}
$$

By using the Brouwer fixed pointed theorem and constructing a suitable Lyapunov function, the authors obtained a set of sufficient conditions for the existence of a globally asymptotically stable periodic solution in system (2). It is well known that the discrete time models are more appropriate than the continuous ones when the size of the population is rarely small or the population has nonoverlapping generations. It has been found that the dynamic behaviors of the discrete system are rather complex and contain more rich dynamics than the continuous ones. To the best of the authors knowledge, still no scholar proposes and studies the discrete predator-prey-mutualist system; this motivated us to study the following system:

$$
\begin{aligned}
& x_{1}(n+1)=x_{1}(n) \exp \left\{a_{1}(n)-b_{1}(n) x_{1}(n)\right. \\
& \left.-\frac{c_{1}(n) x_{3}(n)}{d_{1}(n)+d_{2}(n) x_{2}(n)}\right\}, \\
& x_{2}(n+1)=x_{2}(n) \exp \left\{a_{2}(n)\right. \\
& \left.-\frac{x_{2}(n)}{d_{3}(n)+d_{4}(n) x_{1}(n)}\right\}, \\
& x_{3}(n+1)=x_{3}(n) \exp \left\{-a_{3}(n)\right. \\
& \left.+\frac{k_{1}(n) c_{1}(n) x_{1}(n)}{d_{1}(n)+d_{2}(n) x_{2}(n)}-b_{2}(n) x_{3}(n)\right\},
\end{aligned}
$$

where $x_{1}(n), x_{2}(n)$, and $x_{3}(n)$ are the population sizes of the prey, mutualist, and predator at $n$th generation, respectively, $a_{1}(n)$ and $a_{2}(n)$ are the intrinsic growth rate of prey and mutualist at $n$th generation, $a_{3}(n)$ is the death rate of the predator at $n$th generation, $k_{1}(n)$ is called the conversion rate at $n$th generation, which denotes the fraction of the prey biomass being converted to predator biomass, and $c_{1}(n)$ is the capture rate of the prey at $n$th generation. The sequences of $d_{4}(n), d_{2}(n)$ are the mutualism sequences. We mention here that, in system (3), we consider the density restriction term of predator species $\left(b_{2}(n) z\right)$; such a consideration is needed since the density of any species is restricted by the environment [10]. Here, we assume that $a_{i}(n)(i=1,2,3)$, $b_{j}(n), c_{j}(n)(j=1,2) k_{1}(n)$, and $d_{r}(n)(r=1,2,3,4)$ are all bounded nonnegative sequences. $a_{i}(n)(i=1,2,3), b_{j}(n)(j=$ $1,2)$ are strictly positive sequences. Note that

$$
\begin{aligned}
& x_{1}(n)=x_{1}(0) \exp \sum_{k=1}^{n-1}\left[a_{1}(k)-b_{1}(k) x_{1}(k)\right. \\
& \left.-\frac{c_{1}(k) x_{3}(k)}{d_{1}(k)+d_{2}(k) x_{2}(k)}\right], \\
& x_{2}(n)=x_{2}(0) \exp \sum_{k=1}^{n-1}\left[a_{2}(k)-\frac{x_{2}(k)}{d_{3}(k)+d_{4}(k) x_{1}(k)}\right], \\
& x_{3}(n)=x_{3}(0) \exp \sum_{k=1}^{n-1}\left[-a_{3}(k)+\frac{k_{1}(k) c_{1}(k) x_{1}(k)}{d_{1}(k)+d_{2}(k) x_{2}(k)}\right. \\
& \left.-b_{2}(k) x_{3}(k)\right] .
\end{aligned}
$$

From the point of view of biology, in the sequence, we assume that $x_{1}(0)>0, x_{2}(0)>0, x_{3}(0)>0$, and then from (4), we know that the solutions of system (3) are positive. We use the following notations for any bounded sequence $x(n)$ :

$$
\begin{aligned}
& x^{u}=\sup _{n \in N} x(n), \\
& x^{l}=\inf _{n \in N} x(n) .
\end{aligned}
$$

We arrange the rest of the paper as follows. In Section 2, we establish a permanence result for (3). In Section 3, the sufficient conditions about the uniqueness and global attractivity of the periodic solution of (3) are obtained. In Section 4 , the sufficient conditions about the extinction of predator species and the stability of prey-mutualist species are obtained. Finally, two suitable examples are given to illustrate that the conditions of the main theorem are feasible. We end this paper by a brief discussion.

\section{Permanence}

Theorem 1. Assume the inequalities $k_{1}^{u} c_{1}^{u} B_{1} / d_{1}^{l}-a_{3}^{l}>0$, and every positive solution $\left(x_{1}(n), x_{2}(n), x_{3}(n)\right)$ of system (3) satisfies

$$
\begin{aligned}
& \limsup _{n \rightarrow \infty} x_{1}(n) \leq B_{1}, \\
& \limsup _{n \rightarrow \infty} x_{2}(n) \leq B_{2}, \\
& \limsup _{n \rightarrow \infty} x_{3}(n) \leq B_{3},
\end{aligned}
$$

where

$$
\begin{aligned}
& B_{1}=\frac{\exp \left\{a_{1}^{u}-1\right\}}{b_{1}^{l}}, \\
& B_{2}=\left(d_{3}^{u}+d_{4}^{u} B_{1}\right) \exp \left\{a_{2}^{u}-1\right\}, \\
& B_{3}=\frac{1}{b_{2}^{l}} \exp \left\{-a_{3}^{l}+\frac{k_{1}^{u} c_{1}^{u} B_{1}}{d_{1}^{l}}-1\right\} .
\end{aligned}
$$


Proof. Since $x_{1}(0)>0, x_{2}(0)>0$, and $x_{3}(0)>0$, then $x_{1}(n)>$ $0, x_{2}(n)>0$, and $x_{3}(n)>0$, for $n \geq 0$. We only need to prove that

$$
\limsup _{n \rightarrow \infty} x_{1}(n) \leq B_{1} \text {. }
$$

Since similar result can be shown for $x_{2}(n)$ and $x_{3}(n)$, then (6) follows obviously. We first assume that there exists $l_{0} \in N$ such that $x_{1}\left(l_{0}+1\right) \geq x_{1}\left(l_{0}\right)$. Then

$$
a_{1}\left(l_{0}\right)-b_{1}\left(l_{0}\right) x_{1}\left(l_{0}\right)-\frac{c_{1}\left(l_{0}\right) x_{3}\left(l_{0}\right)}{d_{1}\left(l_{0}\right)+d_{2}\left(l_{0}\right) x_{2}\left(l_{0}\right)} \geq 0 .
$$

Hence,

$$
x_{1}\left(l_{0}\right) \leq \frac{a_{1}\left(l_{0}\right)}{b_{1}\left(l_{0}\right)} \leq \frac{a_{1}^{u}}{b_{1}^{l}} .
$$

It follows that

$$
\begin{aligned}
& x_{1}\left(l_{0}+1\right)=x_{1}\left(l_{0}\right) \exp \left\{a_{1}\left(l_{0}\right)-b_{1}\left(l_{0}\right) x_{1}\left(l_{0}\right)\right. \\
& \left.-\frac{c_{1}\left(l_{0}\right) x_{3}\left(l_{0}\right)}{d_{1}\left(l_{0}\right)+d_{2}\left(l_{0}\right) x_{2}\left(l_{0}\right)}\right\} \leq x_{1}\left(l_{0}\right) \exp \left\{a_{1}\left(l_{0}\right)\right. \\
& \left.-b_{1}\left(l_{0}\right) x_{1}\left(l_{0}\right)\right\} \leq x_{1}\left(l_{0}\right) \exp \left\{a_{1}^{u}-b_{1}^{l} x_{1}\left(l_{0}\right)\right\} \\
& \leq \frac{\exp \left\{a_{1}^{u}-1\right\}}{b_{1}^{l}}=B_{1} ;
\end{aligned}
$$

here we used

$$
\max _{x \in R} x \exp (a-b x)=\frac{\exp (a-1)}{b}, \text { for } a, b>0 .
$$

We claim that

$$
x_{1}(n) \leq B_{1}, \quad n \geq l_{0} .
$$

By way of contradiction, assume that there exists $p_{0}>l_{0}$ such that $x_{1}\left(p_{0}\right)>B_{1}$. Then $p_{0} \geq l_{0}+2$. Let $\widetilde{p_{0}} \geq l_{0}+2$ be the smallest integer such that $x_{1}\left(\widetilde{p_{0}}\right)>B_{1}$. Then $x_{1}\left(\widetilde{p_{0}}\right)>B_{1} \geq x_{1}\left(\widetilde{p_{0}}-\right.$ $1)$, which implies $x_{1}\left(\widetilde{p_{0}}\right)>x_{1}\left(\widetilde{p_{0}}-1\right)$. The above argument produces that $x_{1}\left(\widetilde{p_{0}}\right) \leq B_{1}$, a contradiction. This proves the claim. Now, we assume that $x(n+1)<x(n)$ for all $n \in N$. In particular, $\lim _{n \rightarrow \infty} x(n)$ exists, denoted by $\bar{x}_{1}$. We claim that $\bar{x}_{1} \leq a_{1}^{u} / b_{1}^{l}$. By way of contradiction, assume that $\bar{x}_{1}>a_{1}^{u} / b_{1}^{l}$. Taking limit in the first equation in system (3) gives

$$
\begin{aligned}
& \lim _{n \rightarrow \infty}\left(a_{1}(n)-b_{1}(n) x_{1}(n)-\frac{c_{1}(n) x_{3}(n)}{d_{1}(n)+d_{2}(n) x_{2}(n)}\right) \\
& \quad=0
\end{aligned}
$$

which is a contradiction since

$$
\begin{gathered}
a_{1}(n)-b_{1}(n) x_{1}(n)-\frac{c_{1}(n) x_{3}(n)}{d_{1}(n)+d_{2}(n) x_{2}(n)} \\
\leq a_{1}(n)-b_{1}(n) x_{1}(n) \leq a_{1}^{u}-b_{1}^{l} \bar{x}_{1}<0
\end{gathered}
$$

This proves the claim. Note that

$$
\begin{aligned}
& \exp (x-1)>x \quad(x>0) . \\
& \frac{\exp \left(a_{1}^{u}-1\right)}{b_{1}^{l}}=\frac{a_{1}^{u}}{b_{1}^{l}} \frac{\exp \left(a_{1}^{u}-1\right)}{a_{1}^{u}}>\frac{a_{1}^{u}}{b_{1}^{l}} .
\end{aligned}
$$

It follows that (8) holds. This completes the proof of the main result.

Theorem 2. Assume the inequalities

$$
\begin{aligned}
& \frac{k_{1}^{l} c_{1}^{l} D_{1}}{d_{1}^{u}+d_{2}^{u} B_{2}}-a_{3}^{u}>0, \\
& a_{1}^{l}-\frac{c_{1}^{u} B_{3}}{d_{1}^{l}+d_{2}^{l} D_{2}}>0,
\end{aligned}
$$

where $B_{2}$ and $B_{3}$ are the same as in Theorem 1. Then

$$
\begin{aligned}
& \liminf _{n \rightarrow \infty} x_{1}(n) \geq D_{1}, \\
& \liminf _{n \rightarrow \infty} x_{2}(n) \geq D_{2}, \\
& \liminf _{n \rightarrow \infty} x_{3}(n) \geq D_{3},
\end{aligned}
$$

where

$$
\begin{aligned}
D_{1} & =\frac{a_{1}^{l}\left(d_{1}^{l}+d_{2}^{l} D_{2}\right)-c_{1}^{u} B_{3}}{b_{1}^{u}\left(d_{1}^{l}+d_{2}^{l} D_{2}\right)} \\
& \cdot \exp \left\{a_{1}^{l}-b_{1}^{u} B_{1}-\frac{c_{1}^{u} B_{3}}{d_{1}^{l}+d_{2}^{l} D_{2}}\right\}, \\
D_{2} & =a_{2}^{l} d_{3}^{l} \exp \left\{a_{2}^{l}-\frac{B_{2}}{d_{3}^{l}}\right\}, \\
D_{3} & =\frac{k_{1}^{l} c_{1}^{l} D_{1}-a_{3}^{u}\left(d_{1}^{u}+d_{2}^{u} B_{2}\right)}{\left(d_{1}^{u}+d_{2}^{u} B_{2}\right) b_{2}^{u}} \\
& \cdot \exp \left\{-a_{3}^{u}+\frac{k_{1}^{l} c_{1}^{l} D_{1}}{d_{1}^{u}+d_{2}^{u} B_{2}}-b_{2}^{u} B_{3}\right\} .
\end{aligned}
$$

Proof. We first show that

$$
\liminf _{n \rightarrow \infty} x_{2}(n) \geq D_{2} .
$$

For any $\varepsilon>0$, there exists $n^{*} \in N$ such that

$$
\begin{aligned}
& x_{1}(n) \leq B_{1}+\varepsilon, \\
& x_{2}(n) \leq B_{2}+\varepsilon, \\
& x_{3}(n) \leq B_{3}+\varepsilon,
\end{aligned}
$$


First, we assume that there exists $l_{0} \geq n^{*}$ such that $x_{2}\left(l_{0}+1\right) \leq$ $x_{2}\left(l_{0}\right)$. Note that, for $n \geq l_{0}$,

$$
\begin{aligned}
& x_{2}(n+1) \\
& \quad=x_{2}(n) \exp \left\{a_{2}(n)-\frac{x_{2}(n)}{d_{3}(n)+d_{4}(n) x_{1}(n)}\right\} \\
& \quad \geq x_{2}(n) \exp \left\{a_{2}(n)-\frac{x_{2}(n)}{d_{3}(n)}\right\} \\
& \quad \geq x_{2}(n) \exp \left\{a_{2}^{l}-\frac{x_{2}(n)}{d_{3}^{l}}\right\} .
\end{aligned}
$$

In particular, with $n=l_{0}$, we get

$$
a_{2}^{l}-\frac{x_{2}\left(l_{0}\right)}{d_{3}^{l}} \leq 0
$$

which implies that $x_{2}\left(l_{0}\right) \geq a_{2}^{l} d_{3}^{l}$. Then

$$
\begin{aligned}
& x_{2}\left(l_{0}+1\right) \\
& \quad=x_{2}\left(l_{0}\right) \exp \left\{a_{2}\left(l_{0}\right)-\frac{x_{2}\left(l_{0}\right)}{d_{3}\left(l_{0}\right)+d_{4}\left(l_{0}\right) x_{1}\left(l_{0}\right)}\right\} \\
& \quad \geq x_{2}\left(l_{0}\right) \exp \left\{a_{2}^{l}-\frac{B_{2}+\varepsilon}{d_{3}^{l}}\right\} \\
& \quad \geq a_{2}^{l} d_{3}^{l} \exp \left\{a_{2}^{l}-\frac{B_{2}+\varepsilon}{d_{3}^{l}}\right\} .
\end{aligned}
$$

Let

$$
x_{2 \varepsilon} \stackrel{\text { def }}{=} a_{2}^{l} d_{3}^{l} \exp \left\{a_{2}^{l}-\frac{B_{2}+\varepsilon}{d_{3}^{l}}\right\} \text {. }
$$

We claim that

$$
x_{2}(n) \geq x_{2 \varepsilon} \text { for } n \geq l_{0} \text {. }
$$

By way of contradiction, assume that there exists $p_{0}>l_{0}$ such that $x_{2}\left(p_{0}\right)<x_{2 \epsilon}$. Then $p_{0} \geq l_{0}+2$. Let $\widetilde{p_{0}} \geq l_{0}+2$ be the smallest integer such that $x_{2}\left(\widetilde{p_{0}}\right)<x_{2 \epsilon}$. Then $x_{2}\left(\widetilde{p_{0}}-1\right) \geq$ $x_{2 \epsilon}>x_{2}\left(\widetilde{p_{0}}\right)$, and clearly $x_{2}\left(\widetilde{p_{0}}-1\right)>x_{2}\left(\widetilde{p_{0}}\right)$. The above argument produces that $x_{2}\left(\widetilde{p_{0}}\right) \geq x_{2 \epsilon}$ for all large $n$. Then $\lim _{n \rightarrow \infty} x_{2}(n)$ exists, denoted by $x_{2}$. We claim that $x_{2} \geq a_{2}^{l} d_{3}^{l}$. By way of contradiction, assume that $x_{2}<a_{2}^{l} d_{3}^{l}$. Taking limit in the second equation in system (3) gives

$$
\lim _{n \rightarrow \infty}\left(a_{2}(n)-\frac{x_{2}(n)}{d_{3}(n)+d_{4}(n) x_{1}(n)}\right)=0,
$$

which is a contradiction since

$$
\lim _{n \rightarrow \infty}\left(a_{2}(n)-\frac{x_{2}(n)}{d_{3}(n)+d_{4}(n) x_{1}(n)}\right) \geq a_{2}^{l}-\frac{x_{2}}{\overline{d_{3}^{l}}}
$$

$>0$.
This proves the claim. Note that

$$
\begin{aligned}
\frac{B_{2}}{d_{3}^{l}} & =\frac{d_{3}^{u} \exp \left\{a_{2}^{u}-1\right\}+d_{4}^{u} \exp \left\{a_{1}^{u}+a_{2}^{u}-2\right\} / b_{1}^{l}}{d_{3}^{l}} \\
& \geq \exp \left\{a_{2}^{u}-1\right\}+\frac{d_{4}^{u} \exp \left\{a_{1}^{u}+a_{2}^{u}-2\right\}}{b_{1}^{l} d_{3}^{l}} \\
& \geq a_{2}^{u}+\frac{d_{4}^{u} \exp \left\{a_{1}^{u}+a_{2}^{u}-2\right\}}{b_{1}^{l} d_{3}^{l}} .
\end{aligned}
$$

Clearly, $a_{2}^{l}-B_{2} / d_{3}^{l}<0$, so $D_{2}<a_{2}^{l} d_{3}^{l}$. We can easily see that (19) holds. The proof of the other two inequalities is similar to the above analysis and we omit the detail here. This completes the proof of the main result.

As a direct corollary of Theorems 1 and 2, from the definition of permanence, we have the following.

Theorem 3. Assume that $\left(H_{1}\right)$ holds. Then system (3) is permanent.

It should be noticed that, from the inequality $k_{1}^{l} c_{1}^{l} D_{1} /\left(d_{1}^{u}+\right.$ $\left.d_{2}^{u} B_{2}\right)-a_{3}^{u}>0$ and from the proofs of Theorems 1 and 2, one knows that where $\left(H_{1}\right)$ holds, the set $\left[D_{1}, B_{1}\right] \times\left[D_{2}, B_{2}\right] \times$ $\left[D_{3}, B_{3}\right]$ is an invariant set of system (3).

\section{Existence and Stability of a Periodic Solution}

Due to seasonal effects of weather, temperature, food supply, mating habits, contact with predators, and other resources or physical environmental quantities, we can assume temporal to be cyclic or periodic [26-28]. In this section, we consider system (3) with $a_{i}(n)(i=1,2,3), b_{j}(n), c_{j}(n)(j=1,2)$, $k_{1}(n)$, and $d_{r}(n)(r=1,2,3,4)$ being periodic with a common period. More precisely, we assume that there exists a positive integer $w$ such that, for $n \in N$,

$$
\begin{aligned}
& 0<a_{i}(n+w)=a_{i}(n), \\
& 0<b_{j}(n+w)=b_{j}(n), \\
& 0<c_{j}(n+w)=c_{j}(n), \\
& 0<d_{k}(n+w)=d_{k}(n), \\
& 0<k_{1}(n+w)=k_{1}(n) .
\end{aligned}
$$

Let $B_{i}, D_{i}, i=1,2,3$, be the same as in Theorems 1 and 2. Our first result concerns the existence of a periodic solution.

Theorem 4. Assume that $\left(H_{1}\right)$ holds; then system (3) has $w$ periodic solution, denoted by $\left(\overline{x_{1}}(n), \overline{x_{2}}(n), \overline{x_{3}}(n)\right)$.

Proof. As noted at the end of the last section that $\left[D_{1}, B_{1}\right] \times$ $\left[D_{2}, B_{2}\right] \times\left[D_{3}, B_{3}\right]$ is an invariant set of system (3), thus we can define a mapping $F$ on $\left[D_{1}, B_{1}\right] \times\left[D_{2}, B_{2}\right] \times\left[D_{3}, B_{3}\right]$ by

$$
\begin{aligned}
& F\left(\overline{x_{1}}(0), \overline{x_{2}}(0), \overline{x_{3}}(0)\right)=\left(\overline{x_{1}}(w), \overline{x_{2}}(w), \overline{x_{3}}(w)\right) \\
& \text { for }\left(\overline{x_{1}}(0), \overline{x_{2}}(0), \overline{x_{3}}(0)\right) \in\left[D_{1}, B_{1}\right] \times\left[D_{2}, B_{2}\right] \times\left[D_{3}, B_{3}\right] .
\end{aligned}
$$


Obviously, $F$ depends continuously on $\left(\overline{x_{1}}(0), \overline{x_{2}}(0), \overline{x_{3}}(0)\right)$. Thus, $F$ is continuous and maps the compact set $\left[D_{1}, B_{1}\right] \times$ $\left[D_{2}, B_{2}\right] \times\left[D_{3}, B_{3}\right]$ into itself. Therefore, $F$ has a fixed point $\left(\overline{x_{1}}(n), \overline{x_{2}}(n), \overline{x_{3}}(n)\right)$. It is easy to see that the solution $\left(\overline{x_{1}}(n), \overline{x_{2}}(n), \overline{x_{3}}(n)\right)$ is $w$-periodic solution of system (3). This completes the proof.

Now, under some additional conditions, we study the global stability of the periodic solution obtained in Theorem 4.

Theorem 5. Assume that (29) and $\left(H_{1}\right)$ hold, and

$$
\begin{aligned}
\lambda_{1}= & \max \left\{\left|1-b_{1}^{u} B_{1}\right|,\left|1-b_{1}^{l} D_{1}\right|\right\}+\frac{c_{1}^{u} B_{3} d_{2}^{u} B_{2}}{\left(d_{1}^{l}+d_{2}^{l} D_{2}\right)^{2}} \\
& +\frac{c_{1}^{u} B_{3}}{d_{1}^{l}+d_{2}^{l} D_{2}}<1, \\
\lambda_{2}= & \max \left\{\left|1-\frac{B_{2}}{d_{3}^{l}+d_{4}^{l} D_{1}}\right|,\left|1-\frac{D_{2}}{d_{3}^{u}+d_{4}^{u} B_{1}}\right|\right\} \\
& +\frac{d_{4}^{u} B_{1} B_{2}}{\left(d_{3}^{l}+d_{4}^{l} D_{1}\right)^{2}}<1, \\
\lambda_{3}= & \max \left\{\left|1-b_{2}^{u} B_{3}\right|,\left|1-b_{2}^{l} D_{3}\right|\right\}+\frac{c_{1}^{u} k_{1}^{u} B_{1}}{d_{1}^{l}+d_{2}^{l} D_{2}} \\
& +\frac{c_{1}^{u} k_{1}^{u} B_{1} B_{2} d_{2}^{u}}{\left(d_{1}^{l}+d_{2}^{l} D_{2}\right)^{2}}<1 .
\end{aligned}
$$

Then for every solution $\left(\overline{x_{1}}(n), \overline{x_{2}}(n), \overline{x_{3}}(n)\right)$ of system (3), one has

$$
\begin{aligned}
& \lim _{n \rightarrow \infty}\left(x_{1}(n)-\overline{x_{1}}(n)\right)=0, \\
& \lim _{n \rightarrow \infty}\left(x_{2}(n)-\overline{x_{2}}(n)\right)=0, \\
& \lim _{n \rightarrow \infty}\left(x_{3}(n)-\overline{x_{3}}(n)\right)=0,
\end{aligned}
$$

where $\left(\overline{x_{1}}(n), \overline{x_{2}}(n), \overline{x_{3}}(n)\right)$ is $w$-periodic solution obtained in Theorem 4.

Proof. Let

$$
\begin{aligned}
& x_{1}(n)=\bar{x}_{1}(n) \exp (u(n)), \\
& x_{2}(n)=\bar{x}_{2}(n) \exp (v(n)), \\
& x_{3}(n)=\bar{x}_{31}(n) \exp (z(n)) .
\end{aligned}
$$

Then system (3) is equivalent to

$$
\begin{aligned}
& u(n+1)=u(n)+b_{1}(n) \bar{x}_{1}(n)[1-\exp \{u(n)\}] \\
& +c_{1}(n) \bar{x}_{3}(n) \frac{[1-\exp \{z(n)\}]}{d_{1}(n)+d_{2}(n) x_{2}(n)} \\
& \quad-\frac{c_{1}(n) \bar{x}_{3}(n) \bar{x}_{2}(n) d_{2}(n)[1-\exp \{v(n)\}]}{\left(d_{1}(n)+d_{2}(n) \bar{x}_{2}(n)\right)\left(d_{1}(n)+d_{2}(n) x_{2}(n)\right)},
\end{aligned}
$$

$$
\begin{aligned}
& v(n+1)=v(n)+\frac{\bar{x}_{2}(n)[1-\exp \{v(n)\}]}{d_{3}(n)+d_{4}(n) x_{1}(n)} \\
& -\frac{\bar{x}_{2}(n) d_{4}(n) \bar{x}_{1}(n)[1-\exp \{u(n)\}]}{\left(d_{3}(n)+d_{4}(n) \bar{x}_{1}(n)\right)\left(d_{3}(n)+d_{4}(n) x_{1}(n)\right)}, \\
& z(n+1)=z(n) \\
& -\frac{k_{1}(n) c_{1}(n) \bar{x}_{1}(n)[1-\exp \{u(n)\}]}{d_{1}(n)+d_{2}(n) x_{2}(n)} \\
& +b_{2}(n) \bar{x}_{3}(n)[1-\exp \{z(n)\}] \\
& +\frac{k_{1}(n) c_{1}(n) \bar{x}_{1}(n) \bar{x}_{2}(n) d_{2}(n)[1-\exp \{v(n)\}]}{\left(d_{1}(n)+d_{2}(n) x_{2}(n)\right)\left(d_{1}(n)+d_{2} \bar{x}_{2}(n)\right)} .
\end{aligned}
$$

By using the mean-value theorem, it follows that

$$
\begin{aligned}
& u(n+1)=u(n)\left[1-b_{1}(n) \bar{x}_{1}(n) \exp \left\{\theta_{1}(n) u(n)\right\}\right] \\
& -\frac{c_{1}(n) \bar{x}_{3}(n) z(n) \exp \left\{\theta_{3}(n) z(n)\right\}}{d_{1}(n)+d_{2}(n) x_{2}(n)} \\
& +\frac{c_{1}(n) d_{2}(n) \bar{x}_{2}(n) \bar{x}_{3}(n) \exp \left\{\theta_{2}(n) v(n)\right\} v(n)}{\left(d_{1}(n)+d_{2}(n) x_{2}(n)\right)\left(d_{1}(n)+d_{2}(n) \bar{x}_{2}(n)\right)} \\
& v(n+1)=v(n)\left[1-\frac{\bar{x}_{2}(n) \exp \left\{\theta_{2}(n) v(n)\right\}}{d_{3}(n)+d_{4}(n) x_{1}(n)}\right] \\
& +\frac{d_{4}(n) \bar{x}_{1}(n) \bar{x}_{2}(n) u(n) \exp \left\{\theta_{1}(n) u(n)\right\}}{\left(d_{3}(n)+d_{4}(n) x_{1}(n)\right)\left(d_{3}(n)+d_{4}(n) \bar{x}_{1}(n)\right)}, \\
& z(n+1)=z(n)\left[1-b_{2}(n) \bar{x}_{3}(n) \exp \left\{\theta_{3}(n) z(n)\right\}\right] \\
& +\frac{c_{1}(n) k_{1}(n) \bar{x}_{1}(n) u(n) \exp \left\{\theta_{1}(n) u(n)\right\}}{d_{1}(n)+d_{2}(n) x_{2}(n)} \\
& \quad-\frac{c_{1}(n) k_{1}(n) d_{2}(n) \bar{x}_{1}(n) \bar{x}_{2}(n) \exp \left\{\theta_{2}(n) v(n)\right\} v(n)}{\left(d_{1}(n)+d_{2}(n) x_{2}(n)\right)\left(d_{1}(n)+d_{2}(n) \bar{x}_{2}(n)\right)},
\end{aligned}
$$

where $\left(\theta_{1}(n), \theta_{2}(n), \theta_{3}(n)\right) \in[0,1]$. To complete the proof, it suffices to show that

$$
\lim _{n \rightarrow \infty} u(n)=\lim _{n \rightarrow \infty} v(n)=\lim _{n \rightarrow \infty} z(n)=0
$$

In view of (31), we can choose $\varepsilon>0$ small enough such that

$$
\begin{aligned}
\lambda_{1}^{\epsilon} & =\max \left\{\left|1-b_{1}^{u}\left(B_{1}+\varepsilon\right)\right|,\left|1-b_{1}^{l}\left(D_{1}-\varepsilon\right)\right|\right\} \\
& +\frac{c_{1}^{u}\left(B_{3}+\varepsilon\right) d_{2}^{u}\left(B_{2}+\varepsilon\right)}{\left(d_{1}^{l}+d_{2}^{l}\left(D_{2}-\varepsilon\right)\right)^{2}}+\frac{c_{1}^{u}\left(B_{3}+\varepsilon\right)}{d_{1}^{l}+d_{2}^{l}\left(D_{2}-\varepsilon\right)}<1 \\
\lambda_{2}^{\epsilon} & =\max \left\{\left|1-\frac{\left(B_{2}+\varepsilon\right)}{d_{3}^{l}+d_{4}^{l}\left(D_{1}-\varepsilon\right)}\right|,\right.
\end{aligned}
$$




$$
\begin{gathered}
\left.\left|1-\frac{\left(D_{2}-\varepsilon\right)}{d_{3}^{u}+d_{4}^{u}\left(B_{1}+\varepsilon\right)}\right|\right\}+\frac{d_{4}^{u}\left(B_{1}+\varepsilon\right)\left(B_{2}+\varepsilon\right)}{\left(d_{3}^{l}+d_{4}^{l}\left(D_{1}-\varepsilon\right)\right)^{2}}<1 \\
\lambda_{3}^{\epsilon}=\max \left\{\left|1-b_{2}^{u}\left(B_{3}+\varepsilon\right)\right|,\left|1-b_{2}^{l}\left(D_{3}-\varepsilon\right)\right|\right\} \\
+\frac{c_{1}^{u} k_{1}^{u}\left(B_{1}+\varepsilon\right)}{d_{1}^{l}+d_{2}^{l}\left(D_{2}-\varepsilon\right)}+\frac{c_{1}^{u} k_{1}^{u}\left(B_{1}+\varepsilon\right)\left(B_{2}+\varepsilon\right) d_{2}^{u}}{\left(d_{1}^{l}+d_{2}^{l}\left(D_{2}-\varepsilon\right)\right)^{2}}
\end{gathered}
$$$$
<1 \text {. }
$$

According to Theorems 1 and 2, there exists $n_{0} \in N$ such that

$$
\begin{aligned}
& D_{1}-\varepsilon \leq \bar{x}_{1}(n) \leq B_{1}+\varepsilon, \\
& D_{2}-\varepsilon \leq \bar{x}_{2}(n) \leq B_{2}+\varepsilon, \\
& D_{3}-\varepsilon \leq \bar{x}_{3}(n) \leq B_{3}+\varepsilon \\
& D_{1}-\varepsilon \leq x_{1}(n) \leq B_{1}+\varepsilon, \\
& D_{2}-\varepsilon \leq x_{2}(n) \leq B_{2}+\varepsilon, \\
& D_{3}-\varepsilon \leq x_{3}(n) \leq B_{3}+\varepsilon
\end{aligned}
$$

for $n \geq n_{0}$.

Notice that $\theta_{1}(n) \in[0,1]$ implies that $\bar{x}_{1}(n) \exp \left\{\theta_{1}(n) u(n)\right\}$ lies between $\bar{x}_{1}(n)$ and $x_{1}(n)$. Similarly, $\bar{x}_{2}(n) \exp \left\{\theta_{2}(n) v(n)\right\}$ lies between $\bar{x}_{2}(n)$ and $x_{2}(n)$, and $\bar{x}_{3}(n) \exp \left\{\theta_{3}(n) z(n)\right\}$ lies between $\bar{x}_{3}(n)$ and $x_{3}(n)$. From (35), we get

$$
\begin{aligned}
& |u(n+1)| \leq|u(n)| \max \left\{\left|1-b_{1}^{u}\left(B_{1}+\varepsilon\right)\right|,\right. \\
& \left.\left|1-b_{1}^{l}\left(D_{1}-\varepsilon\right)\right|\right\}+|v(n)| \frac{c_{1}^{u}\left(B_{3}+\varepsilon\right) d_{2}^{u}\left(B_{2}+\varepsilon\right)}{\left(d_{1}^{l}+d_{2}^{l}\left(D_{2}-\varepsilon\right)\right)^{2}} \\
& \quad+|z(n)| \frac{c_{1}^{u}\left(B_{3}+\varepsilon\right)}{d_{1}^{l}+d_{2}^{l}\left(D_{2}-\varepsilon\right)}, \\
& |v(n+1)| \leq|v(n)| \max \left\{\left|1-\frac{B_{2}+\varepsilon}{d_{3}^{l}+d_{4}^{l}\left(D_{1}-\varepsilon\right)}\right|,\right. \\
& \left.\quad\left|1-\frac{D_{2}-\varepsilon}{d_{3}^{u}+d_{4}^{u}\left(B_{1}+\varepsilon\right)}\right|\right\} \\
& \quad+|u(n)| \frac{d_{4}^{u}\left(B_{1}+\varepsilon\right)\left(B_{2}+\varepsilon\right)}{\left(d_{3}^{l}+d_{4}^{l}\left(D_{1}-\varepsilon\right)\right)^{2}}, \\
& \quad+|v(n)| \frac{c_{1}^{u} k_{1}^{u} d_{2}^{u}\left(B_{1}+\varepsilon\right)\left(B_{2}+\varepsilon\right)}{\left(d_{1}^{l}+d_{2}^{l}\left(D_{2}-\varepsilon\right)\right)^{2}} \\
& \left.\left|1-b_{2}^{u}\left(B_{3}+\varepsilon\right)\right|\right\}+|u(n)| \frac{c_{1}^{u} k_{1}^{u}\left(B_{1}+\varepsilon\right)}{d_{1}^{l}+d_{2}^{l}\left(D_{2}-\varepsilon\right)} \\
& +|z(n)| \max \left\{\left|1-b_{2}^{l}\left(D_{3}-\varepsilon\right)\right|,\right.
\end{aligned}
$$

for $n \geq n_{0}$. Let $\lambda=\max \left\{\lambda_{1}^{\varepsilon}, \lambda_{2}^{\varepsilon}, \lambda_{3}^{\varepsilon}\right\}$. Then $\lambda<1$. In view of (39), we get

$$
\begin{aligned}
& \max \{|u(n+1)|,|v(n+1)|,|z(n+1)|\} \\
& \quad \leq \lambda \max \{|u(n)|,|v(n)|,|z(n)|\}, \quad n \geq n_{0} .
\end{aligned}
$$

This implies

$$
\begin{aligned}
& \max \{|u(n)|,|v(n)|,|z(n)|\} \\
& \quad \leq \lambda^{n-n_{0}} \max \left\{\left|u\left(n_{0}\right)\right|,\left|v\left(n_{0}\right)\right|,\left|z\left(n_{0}\right)\right|\right\}, \quad n \geq n_{0} .
\end{aligned}
$$

Therefore (36) holds and the proof is complete.

\section{Extinction of Predator Species and Stability of Prey-Mutualist Species}

In this section, we also consider system (3) with $a_{i}(n)(i=$ $1,2,3), b_{j}(n), c_{j}(n)(j=1,2) k_{1}(n)$, and $d_{r}(n)(r=1,2,3,4)$ being periodic with a common period $w>0$. By developing the analysis technique of [29], we show that, under some suitable assumption, the predator will be driven to extinction while prey-mutualist will be globally attractive to a certain solution of a logistic equation.

We consider a discrete logistic equation

$$
x(n+1)=x(n) \exp \left(a_{1}(n)-b_{1}(n) x(n)\right), \quad n \in N .
$$

Theorem 6. For any positive solution $x^{*}$ of (42), one has

$$
m_{1} \leq \liminf _{n \rightarrow \infty} x^{*} \leq \limsup _{n \rightarrow \infty} x^{*} \leq B_{1}
$$

where $m_{1}=\left(a_{1}^{l} / b_{1}^{u}\right) \exp \left\{a_{1}^{l}-b_{1}^{u} B_{1}\right\}$ and $B_{1}$ is defined by Theorem 1. Furthermore, there exists $w$-periodic solution for (42).

The proof of the above claim follows that of Theorems 1 and 2 with slight modification and we omit the detail here.

Theorem 7. Assume that the inequality

$$
\frac{b_{1}^{u} \exp \left(a_{1}^{u}-1\right)}{b_{1}^{l}}<2
$$

holds. Let $x^{*}(n)$ be a periodic solution of (42). Then, for every positive solution $x(n)$ of (42), one has

$$
\lim _{n \rightarrow \infty}\left(x(n)-x^{*}(n)\right)=0 .
$$

Proof. Let

$$
x(n)=x^{*}(n) \exp \{p(n)\} .
$$

Then system (42) is equivalent to

$$
\begin{aligned}
p(n+1) & =p(n)+b_{1}(n)\left(x^{*}(n)-x(n)\right) \\
& =p(n)-b_{1}(n) x^{*}(n)[\exp p(n)-1] .
\end{aligned}
$$


By using the mean-value theorem, it follows that

$$
p(n+1)=p(n)\left[1-b_{1}(n) x^{*}(n) \exp \left\{\theta_{4}(n) p(n)\right\}\right],
$$

where $\theta_{4}(n) \in[0,1]$. To complete the proof, it suffices to show that

$$
\lim _{n \rightarrow \infty} p(n)=0
$$

we first assume that

$$
\lambda^{*}=\max \left\{\left|1-b_{1}^{u} B_{1}\right|,\left|1-b_{1}^{l} m_{1}\right|\right\}<1 ;
$$

then we can choose positive constant $\varepsilon>0$ small enough such that

$$
\lambda_{\varepsilon}^{*}=\max \left\{\left|1-b_{1}^{u}\left(B_{1}+\varepsilon\right)\right|,\left|1-b_{1}^{l}\left(m_{1}-\varepsilon\right)\right|\right\}<1 .
$$

According to Theorem 6, there exists $n^{*} \in N$ such that

$$
\begin{aligned}
& m_{1}-\varepsilon \leq x(n), \\
& x^{*}(n) \leq B_{1}+\varepsilon, \\
& n \geq n^{*} .
\end{aligned}
$$

Notice that $\theta_{4}(n) \in[0,1]$ implies that $x^{*}(n) \exp \left\{\theta_{4}(n) p(n)\right\}$ lies between $x^{*}(n)$ and $x(n)$. From (47), we get

$$
\begin{aligned}
& |p(n+1)| \\
& \quad \leq|p(n)| \max \left\{\left|1-b_{1}^{u}\left(B_{1}+\varepsilon\right)\right|,\left|1-b_{1}^{l}\left(m_{1}-\varepsilon\right)\right|\right\} \\
& \quad=|p(n)| \lambda_{\varepsilon}^{*} .
\end{aligned}
$$

This implies that

$$
|p(n)| \leq\left(\lambda_{\varepsilon}^{*}\right)^{n-n^{*}}\left|p\left(n^{*}\right)\right| \quad n \geq n^{*}
$$

Since $\lambda_{\varepsilon}^{*}<1$ and $\varepsilon$ is arbitrarily small, we obtain $\lim _{n \rightarrow \infty} p(n)=0$, and it means that (48) holds when $\lambda^{*}<1$.

Note that

$$
1-b_{1}^{u} B_{1} \leq 1-b_{1}^{l} m_{1}<1
$$

thus, $\lambda^{*}<1$ is equivalent to

$$
1-b_{1}^{u} B_{1}>-1
$$

or

$$
b_{1}^{u} B_{1}=\frac{b_{1}^{u}}{b_{1}^{l}} \exp \left\{a_{1}^{u}-1\right\}<2 .
$$

Now, we can conclude that $(48)$ is satisfied as $\left(\mathrm{H}_{2}\right)$ holds, and so

$$
\lim _{n \rightarrow \infty}\left(x(n)-x^{*}(n)\right)=0
$$

Theorem 8. Assume that the inequality

$$
\frac{k_{1}^{u} c_{1}^{u} B_{1}}{d_{1}^{l}+d_{2}^{l} D_{2}}-a_{3}^{l}<0
$$

holds, where $D_{2}$ and $B_{1}$ are defined by Theorems 1 and 2. Let $\left(x_{1}(n), x_{2}(n), x_{3}(n)\right)$ be any positive solution of system (3); then $x_{3}(n) \rightarrow 0$ as $n \rightarrow+\infty$.

Proof. From $\left(\mathrm{H}_{3}\right)$ we can choose positive constant $\varepsilon>0$ small enough such that inequality

$$
\frac{k_{1}^{u} c_{1}^{u}\left(B_{1}+\varepsilon\right)}{d_{1}^{l}+d_{2}^{l}\left(D_{2}-\varepsilon\right)}-a_{3}^{l}<0
$$

holds. Thus, there exists $\delta_{2}>0$,

$$
\frac{k_{1}^{u} c_{1}^{u}\left(B_{1}+\varepsilon\right)}{d_{1}^{l}+d_{2}^{l}\left(D_{2}-\varepsilon\right)}-a_{3}^{l}<-\delta_{2}<0 .
$$

Let $\left(x_{1}(n), x_{2}(n), x_{3}(n)\right)$ be any positive solution of system (3). For any $q \in N$, according to the equation of system (3), we obtain

$$
\begin{aligned}
\ln \frac{x_{3}(q+1)}{x_{3}(q)}= & -a_{3}(q)+\frac{k_{1}(q) c_{1}(q) x_{1}(q)}{d_{1}(q)+d_{2}(q) x_{2}(q)} \\
& -b_{2}(q) x_{3}(q) \\
\leq & -a_{3}(q)+\frac{k_{1}(q) c_{1}(q) x_{1}(q)}{d_{1}(q)+d_{2}(q) x_{2}(q)} \\
\leq & -a_{3}^{l}+\frac{k_{1}^{u} c_{1}^{u}\left(B_{1}+\varepsilon\right)}{d_{1}^{l}+d_{2}^{l}\left(D_{2}-\varepsilon\right)} \leq-\delta_{2}<0 .
\end{aligned}
$$

Summating both sides of the above inequations from 0 to $n-1$, we obtain

$$
\ln \frac{x_{3}(n)}{x_{3}(0)}<-\delta_{2} n,
$$

and then

$$
x_{3}(n)<x_{3}(0) \exp \left(-\delta_{2} n\right) .
$$

The above inequality shows that $x_{3}(n) \rightarrow 0$ exponentially as $n \rightarrow+\infty$. This completes the proof of Theorem 8 .

Theorem 9. Assume $\left(H_{2}\right),\left(H_{3}\right)$, and $B_{2} /\left(d_{3}^{l}+d_{4}^{l} D_{1}\right)<2$ hold; also

$$
\begin{aligned}
a_{1}^{l}-\frac{c_{1}^{u} B_{3}}{d_{1}^{l}+d_{2}^{l} D_{2}}>0, \\
\frac{k_{1}^{u} c_{1}^{u} B_{1}}{d_{1}^{l}}-a_{3}^{l}>0 .
\end{aligned}
$$

Then for any positive solution $\left(x_{1}(n), x_{2}(n), x_{3}(n)\right)$ of system (3), one has

$$
\begin{aligned}
& \lim _{n \rightarrow \infty}\left(x_{1}(n)-x_{1}^{*}(n)\right)=0, \\
& \lim _{n \rightarrow \infty}\left(x_{2}(n)-x_{2}^{*}(n)\right)=0 .
\end{aligned}
$$

$x_{1}^{*}(n)$ is any positive solution of system (42) and $x_{2}^{*}(n)$ is any positive solution of the second equation of system (3). 
Proof. Since $\left(\mathrm{H}_{3}\right)$ holds, it follows from Theorem 8 that

$$
\lim _{n \rightarrow \infty} x_{3}(n)=0
$$

To prove $\lim _{n \rightarrow \infty}\left(x_{1}(n)-x_{1}^{*}(n)\right)=0$, let

$$
x_{1}(n)=x_{1}^{*}(n) \exp (u(n)) ;
$$

then from the first equation of system (3) and (66),

$$
\begin{aligned}
u(n+1)= & u(n)-b_{1}(n) x_{1}^{*}(n)(\exp (u(n)-1)) \\
& -\frac{c_{1}(n) x_{3}(n)}{d_{1}(n)+d_{2}(n) x_{2}(n)} .
\end{aligned}
$$

Using the mean-value theorem, one has

$$
\begin{aligned}
& \exp (u(n)-1)=\exp \left(\zeta_{1}(n) u(n)\right) u(n), \\
& \qquad \zeta_{1}(n) \in(0,1) .
\end{aligned}
$$

Then the first equation of system (3) is equivalent to

$$
\begin{aligned}
u(n+1)= & u(n)\left(1-b_{1}(n) x_{1}^{*}(n) \exp \left(\zeta_{1}(n) u(n)\right)\right) \\
& -\frac{c_{1}(n) x_{3}(n)}{d_{1}(n)+d_{2}(n) x_{2}(n)} .
\end{aligned}
$$

To complete the proof, it suffices to show that

$$
\lim _{n \rightarrow \infty} u(n)=0
$$

We first assume that

$$
\lambda=\max \left\{\left|1-b_{1}^{u} B_{1}\right|,\left|1-b_{1}^{l} D_{1}\right|\right\}<1,
$$

and then we can choose positive constant $\varepsilon>0$ small enough such that

$$
\lambda_{\varepsilon}=\max \left\{\left|1-b_{1}^{u}\left(B_{1}+\varepsilon\right)\right|,\left|1-b_{1}^{l}\left(D_{1}-\varepsilon\right)\right|\right\}<1 .
$$

For the above $\varepsilon$, according to Theorems 1, 2, and 8, there exists an integer $n_{1} \in N$ such that

$$
\begin{aligned}
& D_{1}-\varepsilon \leq x_{1}(n) \leq B_{1}+\varepsilon, \\
& m_{1}-\varepsilon \leq x_{1}^{*}(n) \leq B_{1}+\varepsilon, \\
& x_{3}(n) \leq \varepsilon,
\end{aligned}
$$

$$
n \geq n_{1}
$$

Noting that $m_{1} \geq D_{1}$, then

$$
\begin{aligned}
& D_{1}-\varepsilon \leq x_{1}(n), \\
& x_{1}^{*}(n) \leq B_{1}+\varepsilon, \\
& x_{3}(n) \leq \varepsilon,
\end{aligned}
$$

It follows from (74) that

$$
\frac{c_{1}(n)}{d_{1}(n)+d_{2}(n) x_{2}(n)} \leq \frac{c_{1}^{u}}{d_{1}^{l}+d_{2}^{l}\left(D_{2}-\varepsilon\right)} \doteq M_{\varepsilon}
$$

$$
n \geq n_{1} \text {. }
$$

Noting that $\zeta_{1}(n) \in(0,1)$, it implies that $x_{1}^{*}(n) \exp \left(\zeta_{1}(n) u(n)\right)$ lies between $x_{1}^{*}(n)$ and $x_{1}(n)$. From (69), (72)-(75), we get

$$
\begin{aligned}
& |u(n+1)| \\
& \leq|u(n)| \max \left\{\left|1-b_{1}^{u}\left(B_{1}+\varepsilon\right)\right|,\left|1-b_{1}^{l}\left(D_{1}-\varepsilon\right)\right|\right\} \\
& \quad+\frac{c_{1}^{u} \varepsilon}{d_{1}^{l}+d_{2}^{l}\left(D_{2}-\varepsilon\right)}=|u(n)| \lambda_{\varepsilon}+M_{\varepsilon} \varepsilon,
\end{aligned}
$$

$$
n \geq n_{1}
$$

This implies that

$$
|u(n)| \leq \lambda_{\varepsilon}^{n-n_{1}}\left|u\left(n_{1}\right)\right|+\frac{1-\lambda_{\varepsilon}^{n-n_{1}}}{1-\lambda_{\varepsilon}} M_{\varepsilon} \varepsilon, \quad n \geq n_{1} .
$$

Since $\lambda_{\varepsilon}<1$ and $\varepsilon$ is arbitrarily small, we obtain $\lim _{n \rightarrow \infty} u(n)=0$, and it means that (70) holds when $\lambda<1$.

Note that

$$
1-b_{1}^{u} B_{1} \leq 1-b_{1}^{l} D_{1}<1
$$

thus, $\lambda<1$ is equivalent to

$$
1-b_{1}^{u} B_{1}>-1
$$

or

$$
b_{1}^{u} B_{1}=\frac{b_{1}^{u}}{b_{1}^{l}} \exp \left\{a_{1}^{u}-1\right\}<2 .
$$

Now, we can conclude that (70) is satisfied as $\left(\mathrm{H}_{2}\right)$ holds, and so

$$
\lim _{n \rightarrow \infty}\left(x_{1}(n)-x_{1}^{*}(n)\right)=0 .
$$

Next, we prove

$$
\lim _{n \rightarrow \infty}\left(x_{2}(n)-x_{2}^{*}(n)\right)=0 .
$$

Let

$$
x_{2}(n)=x_{2}^{*}(n) \exp (v(n)) .
$$

If $k_{1}^{u} c_{1}^{u} B_{1} / d_{1}^{l}-a_{3}^{l}>0$ and $a_{1}^{l}-c_{1}^{u} B_{3} /\left(d_{1}^{l}+d_{2}^{l} D_{2}\right)>0$ hold, from Theorems 1 and 2 , we know that $x_{1}(n), x_{2}(n)$ are bounded eventually. From the second inequality of (39),

$$
|v(n+1)| \leq|v(n)| \max \left\{\left|1-\frac{B_{2}+\varepsilon}{d_{3}^{l}+d_{4}^{l}\left(D_{1}-\varepsilon\right)}\right|,\right.
$$

$$
\begin{aligned}
& \left.\left|1-\frac{D_{2}-\varepsilon}{d_{3}^{u}+d_{4}^{u}\left(B_{1}+\varepsilon\right)}\right|\right\} \\
& +|u(n)| \frac{d_{4}^{u}\left(B_{1}+\varepsilon\right)\left(B_{2}+\varepsilon\right)}{\left(d_{3}^{l}+d_{4}^{l}\left(D_{1}-\varepsilon\right)\right)^{2}}
\end{aligned}
$$


We first assume that

$$
\rho=\max \left\{\left|1-\frac{B_{2}}{d_{3}^{l}+d_{4}^{l} D_{1}}\right|,\left|1-\frac{D_{2}}{d_{3}^{u}+d_{4}^{u} B_{1}}\right|\right\}<1 .
$$

It follows from (70) that $\lim _{n \rightarrow \infty} u(n)=0$.

For any positive constant $\varepsilon>0$, there exists integer $n_{2} \geq$ $\max \left(n_{0}, n_{1}\right)$ such that

$$
\begin{aligned}
& \rho_{\varepsilon}=\max \left\{\left|1-\frac{\left(B_{2}+\varepsilon\right)}{d_{3}^{l}+d_{4}^{l}\left(D_{1}-\varepsilon\right)}\right|,\right. \\
& \left.\left|1-\frac{\left(D_{2}-\varepsilon\right)}{d_{3}^{u}+d_{4}^{u}\left(B_{1}+\varepsilon\right)}\right|\right\}<1, \quad n \geq n_{2} \\
& |u(n)|<\varepsilon, \quad n \geq n_{2} .
\end{aligned}
$$

Let

$$
\frac{d_{4}^{u}\left(B_{1}+\varepsilon\right)\left(B_{2}+\varepsilon\right)}{\left(d_{3}^{l}+d_{4}^{l}\left(D_{1}-\varepsilon\right)\right)^{2}} \doteq A_{\varepsilon} .
$$

From (84)-(87) we can conclude that

$$
|v(n+1)| \leq \rho_{\varepsilon}|v(n)|+\varepsilon A_{\varepsilon}, \quad n \geq n_{2} .
$$

This implies that

$$
|v(n)| \leq \rho_{\varepsilon}^{n-n_{2}}\left|v\left(n_{2}\right)\right|+\varepsilon A_{\varepsilon} \frac{1-\rho_{\varepsilon}^{n-n_{2}}}{1-\rho_{\varepsilon}}, \quad n \geq n_{2} .
$$

Since $\rho_{\varepsilon}<1$ and $\varepsilon$ is arbitrarily small, we obtain $\lim _{n \rightarrow \infty} u(n)=0$. Note that

$$
1-\frac{B_{2}}{d_{3}^{l}+d_{4}^{l} D_{1}} \leq 1-\frac{D_{2}}{d_{3}^{u}+d_{4}^{u} B_{1}}<1
$$

thus $\rho_{\varepsilon}<1$ is equivalent to

$$
1-\frac{B_{2}}{d_{3}^{l}+d_{4}^{l} D_{1}}>-1,
$$

or

$$
\frac{B_{2}}{d_{3}^{l}+d_{4}^{l} D_{1}}<2
$$

Now, we can conclude that $\lim _{n \rightarrow \infty} v(n)=0$. And so

$$
\lim _{n \rightarrow \infty}\left(x_{2}(n)-x_{2}^{*}(n)\right)=0 .
$$

We can conclude that

$$
\begin{aligned}
& \lim _{n \rightarrow \infty}\left(x_{1}(n)-x_{1}^{*}(n)\right)=0, \\
& \lim _{n \rightarrow \infty}\left(x_{2}(n)-x_{2}^{*}(n)\right)=0, \\
& n \geq n_{2}
\end{aligned}
$$

This completes the proof of Theorem 9.

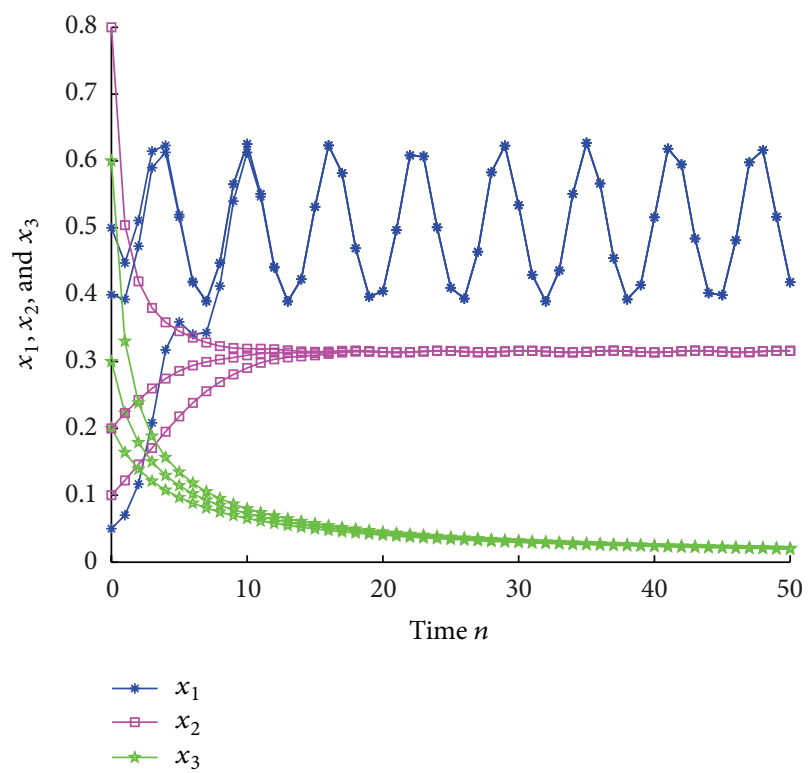

FIgURE 1: Dynamic behavior of the solution $\left(x_{1}(n), x_{2}(n), x_{3}(n)\right)$ to system (95) with the initial conditions $\left(x_{1}(0), x_{2}(0), x_{3}(0)\right)=$ $(0.05,0.1,0.2),(0.5,0.8,0.3)$ and $(0.4,0.2,0.6)$, respectively.

\section{Examples and Numeric Simulations}

In this section, we will give two examples to show the feasibility of our results.

Example 1. Consider the following system:

$$
\begin{aligned}
& x_{1}(n+1)=x_{1}(n) \\
& \cdot \exp \left\{(0.5-0.2 \cos (n))-x_{1}(n)-\frac{0.02 x_{3}(n)}{1+x_{2}(n)}\right\}, \\
& x_{2}(n+1)=x_{2}(n) \exp \left\{0.3-\frac{x_{2}(n)}{1+0.1 x_{1}(n)}\right\} \\
& x_{3}(n+1)=x_{3}(n) \\
& \quad \cdot \exp \left\{-0.0001+\frac{0.01 x_{1}(n)}{1+x_{2}(n)}-x_{3}(n)\right\}
\end{aligned}
$$

One could easily see that $k_{1}^{l} c_{1}^{l} D_{1} /\left(d_{1}^{u}+d_{2}^{u} B_{2}\right)-a_{3}^{u} \approx$ $0.0011>0$ and $a_{1}^{l}-c_{1}^{u} B_{3} /\left(d_{1}^{l}+d_{2}^{l} D_{2}\right) \approx 0.2940>0$, and then condition $\left(H_{1}\right)$ is satisfied. According to Theorem 1 , system (3) is permanent. Numerical simulation (see Figure 1) indicates the permanence of system (95).

Figure 1 shows the dynamic behaviors of system (95), which strongly supports our results.

Example 2. Consider the following system:

$$
\begin{aligned}
& x_{1}(n+1)=x_{1}(n) \\
& \cdot \exp \left\{(0.7-0.3 \cos (n))-2 x_{1}(n)-\frac{0.02 x_{3}(n)}{1+x_{2}(n)}\right\},
\end{aligned}
$$




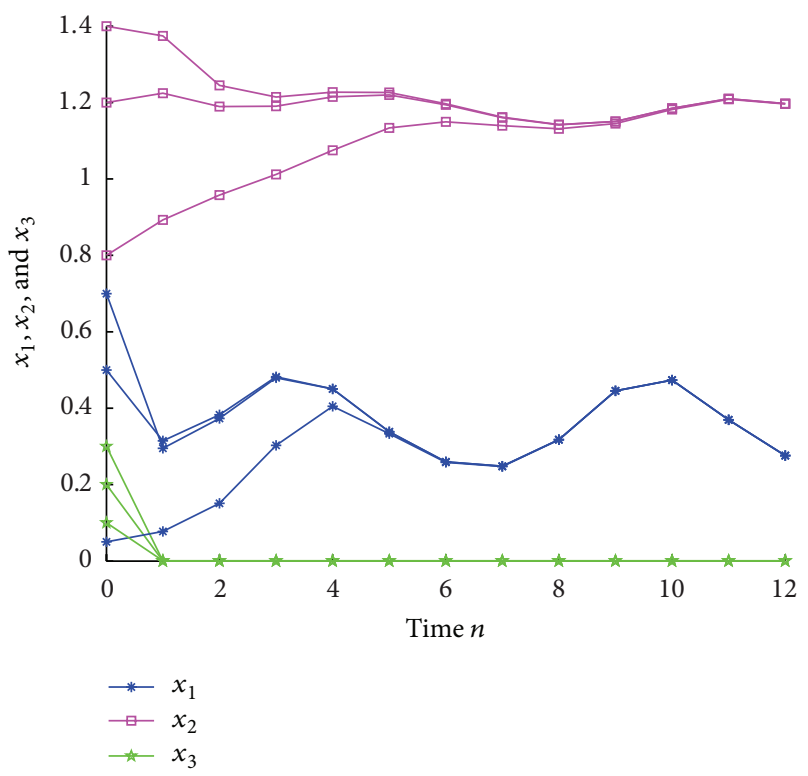

Figure 2: Dynamic behavior of the solution $\left(x_{1}(n), x_{2}(n), x_{3}(n)\right)$ to system (96) with the initial conditions $\left(x_{1}(0), x_{2}(0), x_{3}(0)\right)=$ $(0.05,0.8,0.1),(0.5,1.2,0.2)$ and $(0.7,1.4,0.3)$, respectively.

$$
\begin{aligned}
& x_{2}(n+1)=x_{2}(n) \\
& \cdot \exp \left\{(0.5-0.1 \sin (n))-\frac{x_{2}(n)}{2+x_{1}(n)}\right\}, \\
& x_{3}(n+1)=x_{3}(n) \\
& \quad \cdot \exp \left\{-0.004+\frac{0.01 x_{1}(n)}{1+x_{2}(n)}-50 x_{3}(n)\right\} .
\end{aligned}
$$

We could easily see that $\left(b_{1}^{u} / b_{1}^{l}\right) \exp \left(a_{1}^{u}-1\right)=1<2$, $k_{1}^{u} c_{1}^{u} B_{1} /\left(d_{1}^{l}+d_{2}^{l} D_{2}\right)-a_{3}^{l} \approx-0.000703<0, B_{2} /\left(d_{3}^{l}+\right.$ $\left.d_{4}^{l} D_{1}\right) \approx 0.7943<2, a_{1}^{l}-c_{1}^{u} B_{3} /\left(d_{1}^{l}+d_{2}^{l} D_{2}\right) \approx 0.3999>0$, and $k_{1}^{u} c_{1}^{u} B_{1} / d_{1}^{l}-a_{3}^{l}=0.001>0$. Clearly, conditions of Theorem 9 are satisfied, and so $\lim _{n \rightarrow \infty}\left(x_{1}(n)-x_{1}^{*}(n)\right)=0$, $\lim _{n \rightarrow \infty}\left(x_{2}(n)-x_{2}^{*}(n)\right)=0$, and $\lim _{n \rightarrow \infty} x_{3}(n)=0$.

Figure 2 shows the dynamic behaviors of system (96), which strongly supports our results.

\section{Discussion}

It is well known that prey-mutualist system can decrease predation risk; mutualism plays an important role in the dynamic behaviors of predator-prey populations. For system (3), we showed that the predator-prey-mutualist system will be coexistent in a globally stable state under some suitable conditions. We argued that it is an important topic to study the extinction of the species [29-32], since, with the development of modern society, more and more species are driven to extinction; this motivated us to study the extinction of the predator species. In Section 4, our results indicate that if the death rate of the predator species $x_{3}$ is big enough or the cooperate effect between species $x_{1}$ and $x_{2}$ is very strong, the predator species will be driven to extinction due to the fewer chances of meeting prey species. This can also be seen from $\left(H_{3}\right) ; x_{3}$ will be driven to extinction when $d_{2}$ becomes bigger.

\section{Conflict of Interests}

The authors declare that there is no conflict of interests regarding the publication of this paper.

\section{Acknowledgments}

The authors are grateful to anonymous referees for their excellent suggestions, which greatly improve the presentation of the paper. Also, the research was supported by the Natural Science Foundation of Fujian Province (2015J01012, 2015J01019) and the Foundation of Fujian Education Bureau.

\section{References}

[1] A. A. Berryman, "The origins and evolution of predator-prey theory," Ecology, vol. 73, no. 5, pp. 1530-1535, 1992.

[2] L. J. Chen, F. D. Chen, and L. J. Chen, "Qualitative analysis of a predator-prey model with Holling type II functional response incorporating a constant prey refuge," Nonlinear Analysis: Real World Applications, vol. 11, no. 1, pp. 246-252, 2010.

[3] Z. Z. Ma, F. D. Chen, C. Q. Wu, and W. L. Chen, "Dynamic behaviors of a Lotka-Volterra predator-prey model incorporating a prey refuge and predator mutual interference," Applied Mathematics and Computation, vol. 219, no. 15, pp. 7945-7953, 2013.

[4] L. J. Chen, F. D. Chen, and Y. Q. Wang, "Influence of predator mutual interference and prey refuge on Lotka-Volterra predator-prey dynamics," Communications in Nonlinear Science and Numerical Simulation, vol. 18, no. 11, pp. 3174-3180, 2013.

[5] L.-L. Wang and W.-T. Li, "Periodic solutions and permanence for a delayed nonautonomous ratio-dependent predator-prey model with Holling type functional response," Journal of Computational and Applied Mathematics, vol. 162, no. 2, pp. 341-357, 2004.

[6] F. D. Chen, Z. Z. Ma, and H. Y. Zhang, "Global asymptotical stability of the positive equilibrium of the Lotka-Volterra preypredator model incorporating a constant number of prey refuges," Nonlinear Analysis: Real World Applications, vol. 13, no. 6, pp. 2790-2793, 2012.

[7] Y. M. Chen and Z. Zhou, "Stable periodic solution of a discrete periodic Lotka-Volterra competition system," Journal of Mathematical Analysis and Applications, vol. 277, no. 1, pp. 358366, 2003.

[8] N. Krikorian, "The Volterra model for three species predatorprey systems: boundedness and stability," Journal of Mathematical Biology, vol. 7, no. 2, pp. 117-132, 1979.

[9] S. W. Zhang and D. J. Tan, "Study for three species model with Holling II founctional response and priodic coefficients," Journal of Biomathematics, vol. 15, pp. 353-357, 2000 (Chinese).

[10] P. Y. H. Pang and M. X. Wang, "Strategy and stationary pattern in a three-species predator-prey model," Journal of Differential Equations, vol. 200, no. 2, pp. 245-273, 2004.

[11] M. Liao, X. Tang, and C. Xu, "Bifurcation analysis for a threespecies predator-prey system with two delays," Communications 
in Nonlinear Science and Numerical Simulation, vol. 17, no. 1, pp. 183-194, 2012.

[12] R. M. May and W. J. Leonard, "Nonlinear aspects of competition between three species," SIAM Journal on Applied Mathematics, vol. 29, no. 2, pp. 243-253, 1975.

[13] K. Das, K. S. Reddy, M. N. Srinivas, and N. H. Gazi, "Chaotic dynamics of a three species prey-predator competition model with noise in ecology," Applied Mathematics and Computation, vol. 231, pp. 117-133, 2014.

[14] B. L. Bentley, "Extrafloral nectaries and protection by pugnacious bodyguards," Annual Review of Ecology and Systematics, vol. 8, no. 1, pp. 407-427, 1977.

[15] L. J. Chen, L. J. Chen, and Z. Li, "Permanence of a delayed discrete mutualism model with feedback controls," Mathematical and Computer Modelling, vol. 50, no. 7-8, pp. 1083-1089, 2009.

[16] Y. F. Chen, R. Y. Han, L. Y. Yang, and F. D. Chen, "Global asymptotical stability of an obligate Lotka-Volterra mutualism model," Annals of Differential Equations, vol. 30, no. 3, pp. 267271, 2014.

[17] F.-D. Chen, J.-L. Shi, and X.-X. Chen, "Periodicity in a LotkaVolterra facultative mutualism system with several delays," Chinese Journal of Engineering Mathematics, vol. 21, no. 3, pp. 403-409, 2004.

[18] L. J. Chen and L. J. Chen, "Permanence of a discrete periodic volterra model with mutual interference," Discrete Dynamics in Nature and Society, vol. 2009, Article ID 205481, 9 pages, 2009.

[19] Y. S. Wang, H. Wu, and S. Sun, "Persistence of pollination mutualisms in plant-pollinator-robber systems," Theoretical Population Biology, vol. 81, no. 3, pp. 243-250, 2012.

[20] Z. Zhang, J. Wu, and Z. Wang, "Periodic solutions of nonautonomous stage-structured cooperative system," Computers and Mathematics with Applications, vol. 47, no. 4-5, pp. 699-706, 2004.

[21] X. D. Xie, F. D. Chen, K. Yang, and Y. L. Xue, "Global attractivity of an integrodifferential model of mutualism," Abstract and Applied Analysis, vol. 2014, Article ID 928726, 6 pages, 2014.

[22] J. F. Addicott, "A multispecies aphid-ant association: density dependence and species-specific effects," Canadian Journal of Zoology, vol. 57, no. 3, pp. 558-569, 1979.

[23] M. J. Way, "Mutualism between ants and honeydew-producing homoptera," Annual Review of Entomology, vol. 8, no. 1, pp. 307344, 1963.

[24] B. Rai and W. Krawcewicz, "Hopf bifurcation in symmetric configuration of predator-prey-mutualist systems," Nonlinear Analysis, Theory, Methods and Applications, vol. 71, no. 9, pp. 4279-4296, 2009.

[25] L. Y. Yang, X. X. Xie, and C. Q. Wu, "Periodic solution of a periodic predator-prey-mutualist system," Communications in Mathematical Biology and Neuroscience, vol. 2015, article 7, 2015.

[26] K. Gopalsamy, "Global asymptotic stability in a periodic LotkaVolterra system," The Journal of the Australian Mathematical Society-Series B: Applied Mathematics, vol. 27, no. 1, pp. 66-72, 1985.

[27] Z. Q. Liang and L. S. Chen, "Stability of periodic solutions for a discrete Leslie predator-prey system," Acta Mathematica Scientia, vol. 26, no. 4, pp. 634-640, 2006 (Chinese).

[28] G. M. Xu and X. H. Chen, "Persistence and periodic solution for three interacting predator-prey system with refuges," Yinshan Academic Journal, vol. 23, no. 1, pp. 14-17, 2009 (Chinese).

[29] Y. M. Wu, F. D. Chen, W. L. Chen, and Y. H. Lin, "Dynamic behaviors of a nonautonomous discrete predator-prey system incorporating a prey refuge and Holling type II functional response," Discrete Dynamics in Nature and Society, vol. 2012, Article ID 508962, 14 pages, 2012.

[30] Z. Li and F. D. Chen, "Extinction in two dimensional discrete Lotka-Volterra competitive system with the effect of toxic substances," Dynamics of Continuous, Discrete and Impulsive Systems B: Applications and Algorithms, vol. 15, no. 2, pp. 165$178,2008$.

[31] F. D. Chen and M. S. You, "Permanence, extinction and periodic solution of the predator-prey system with BeddingtonDeAngelis functional response and stage structure for prey," Nonlinear Analysis: Real World Applications, vol. 9, no. 2, pp. 207-221, 2008.

[32] Z. Li and F. D. Chen, "Extinction in two dimensional nonautonomous Lotka-Volterra systems with the effect of toxic substances," Applied Mathematics and Computation, vol. 182, no. 1, pp. 684-690, 2006. 


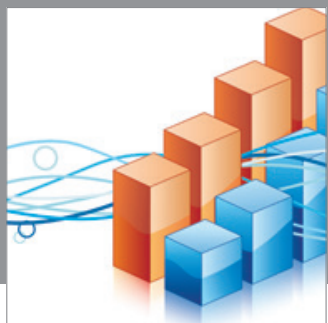

Advances in

Operations Research

mansans

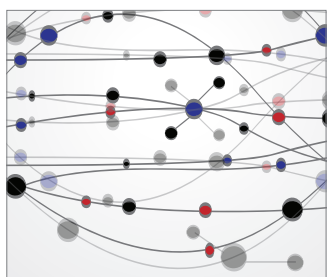

The Scientific World Journal
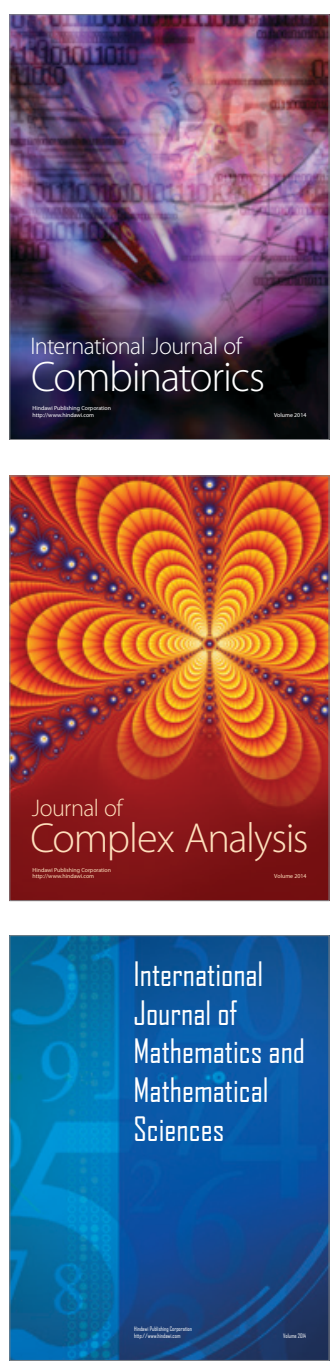
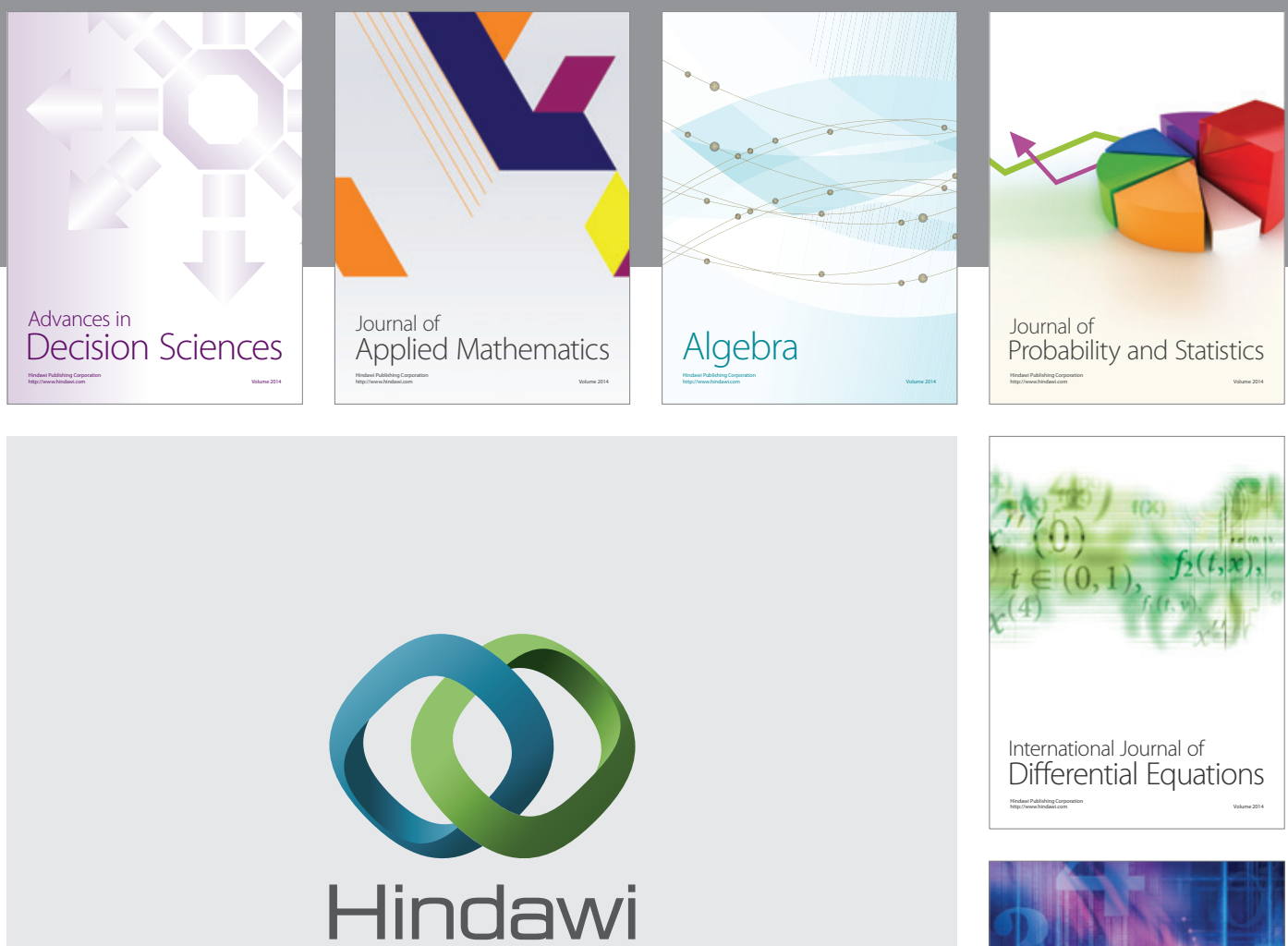

Submit your manuscripts at http://www.hindawi.com
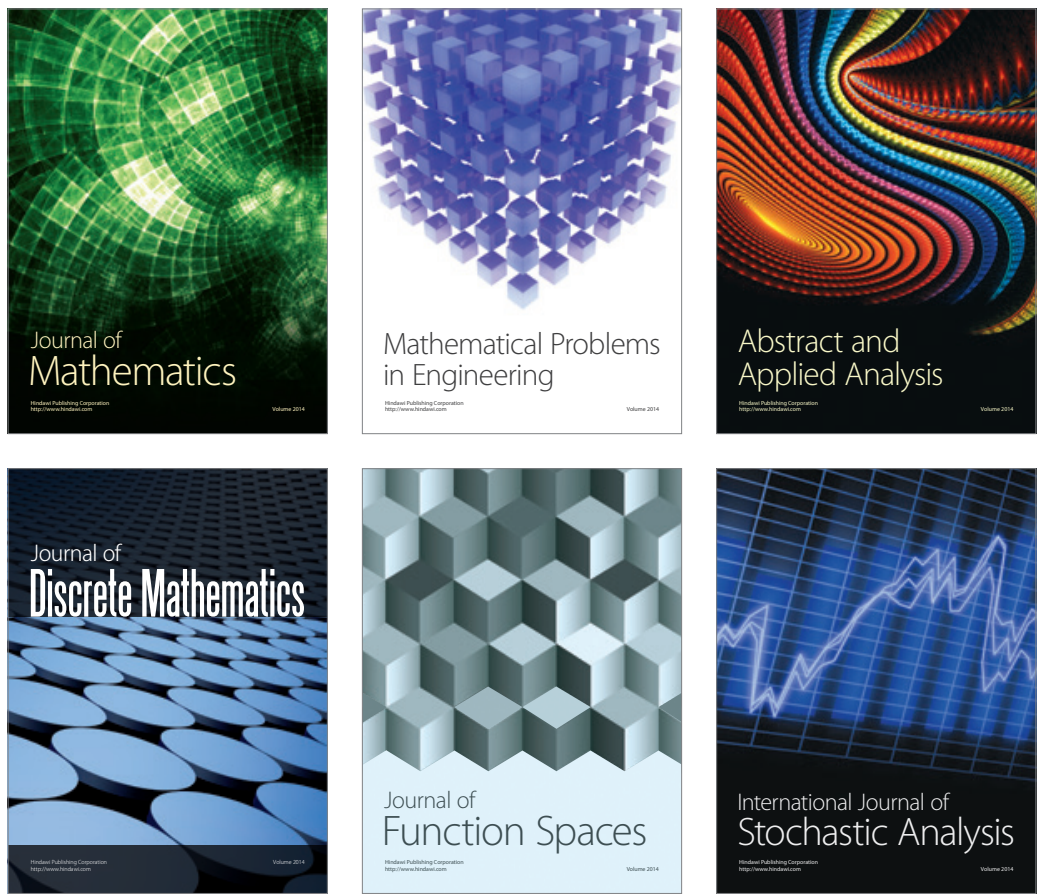

Journal of

Function Spaces

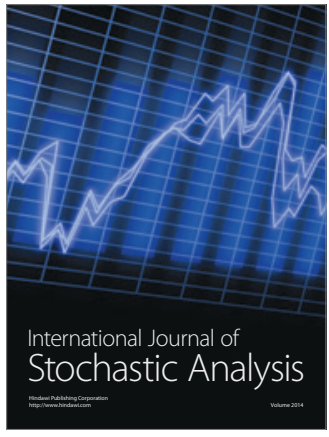

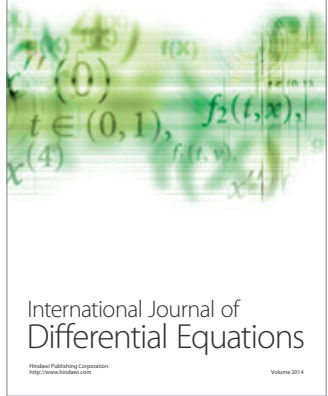
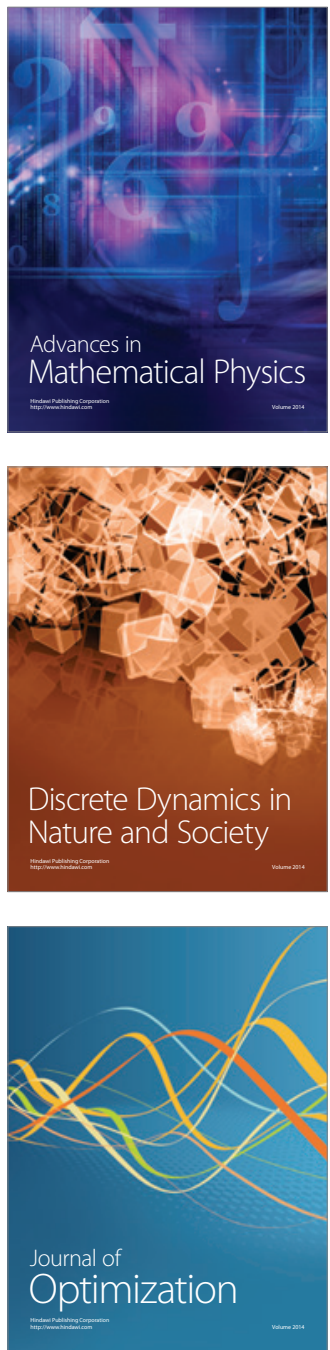